\title{
The Origins of Independence Algebras
}

\begin{abstract}
In this paper we describe how independence algebras could have been discovered and how $v^{*}$-algebras probably were discovered. We also provide a set of equivalent definitions for independence algebras (and hence for $v^{*}$-algebras) and mention some of the uses of these algebras in semigroup theory.
\end{abstract}

Mathematics subject classification: 20M20.

\section{A Heuristic Approach to Independence Algebras}

Let $S$ be a monoid (that is, a semigroup with identity). We say that two elements $a, b \in S$ are $\mathcal{J}$-related if $S a S=S b S$. It is well known (see [26]) that $\mathcal{J}$ is an equivalence relation and that we can introduce a partial order in the set $S / \mathcal{J}$ of $\mathcal{J}$-classes as follows: for all $x, y \in S$,

$$
J_{x} \leqslant \mathcal{J} J_{y} \Leftrightarrow x \in S y S .
$$

Now suppose that we have proved an interesting theorem about semigroups in which $\left(S / \mathcal{J}, \leqslant_{\mathcal{J}}\right)$ is a well ordered chain. Clearly our next aim would be to provide natural examples of semigroups with this property.

When $S=T(X)$, the transformation monoid on a set $X$, then

$$
\left(\forall_{x, y \in S}\right) x \in S y S \Leftrightarrow|\operatorname{im}(x)| \leqslant|\operatorname{im}(y)| .
$$

Similarly, when $S=\operatorname{End}(V)$, the endomorphism monoid of a vector space $V$, we have

$$
\left(\forall_{x, y \in S}\right) x \in S y S \Leftrightarrow \operatorname{rank}(x) \leqslant \operatorname{rank}(y),
$$

where $\operatorname{rank}(x)$ is the dimension of $\operatorname{im}(x)$.

For both $S=T(X)$ and $S=\operatorname{End}(V)$ one of $J_{x} \leqslant \mathcal{J} J_{y}$ or $J_{y} \leqslant \mathcal{J} J_{x}$ holds for all $x, y \in S$. Thus these are two examples of monoids in which $(S / \mathcal{J}, \leqslant \mathcal{J})$ is a well ordered chain.

Following this path, one may observe, considering the two examples above, that $V$ is a universal algebra and $\operatorname{End}(V)$ is the monoid of all mappings $f: V \rightarrow V$ which preserve the operations of the algebra $V$. In the same way, the set $X$ is a universal algebra (with no operations) and $T(X)$ is 
the monoid of all mappings $f: X \rightarrow X$ that preserve the operations of the algebra $X$. Thus a natural monoid to be considered is $\operatorname{End}(\mathcal{A})$, where $\mathcal{A}$ is a universal algebra. This is not only a natural example of a monoid, but, in fact, every monoid is isomorphic to $\operatorname{End}(\mathcal{A})$, for some algebra $\mathcal{A}$. (See, for example, [20] or [22].) Clearly then, we need to impose some conditions on our algebra if its endomorphism monoid is to have the property we desire.

For an element $a \in \operatorname{End}(\mathcal{A})$, we denote the image of $a$ by $\operatorname{im}(a)$. Now let $a, b \in S=\operatorname{End}(\mathcal{A})$, and suppose, by analogy with the two examples above, that we could prove that $a \in S b S$ if and only the dimension of the image of $a$ (in short, $\operatorname{dim}(\operatorname{im}(a)))$ is less than or equal to the dimension of the image of $b$, that is, suppose we were able to prove something of the form

$$
a \in S b S \Leftrightarrow \operatorname{dim}(\operatorname{im}(a)) \leqslant \operatorname{dim}(\operatorname{im}(b)) .
$$

If such a proposition were true, then $S$ would be an example of a monoid in which $\left(S / \mathcal{J}, \leqslant_{\mathcal{J}}\right)$ is a chain. The problem here is that $\operatorname{dim}(\operatorname{im}(a))$ might not be defined. Next we consider how we might obtain a well defined notion of dimension.

We say that $B$ is a basis for $\operatorname{im}(a)$ if $B$ is a minimal generating set for $\operatorname{im}(a)$, that is,

$$
\begin{aligned}
& \text { (1) } B \subseteq \operatorname{im}(a) ; \\
& \text { (2) }\langle B\rangle=\operatorname{im}(a) ; \\
& \text { (3) }\left(\forall_{b \in B}\right)\langle B \backslash b\rangle \varsubsetneqq \operatorname{im}(a) .
\end{aligned}
$$

A finite algebra always has a basis. However, two bases of an algebra might have different cardinalities and hence for that algebra we cannot define the dimension (i.e., the number of elements of any basis).

Thus, in order to be able to speak about dimension of an algebra, we must restrict our study to those algebras which have a basis and in which all the bases have the same cardinality. It is well known (in matroid theory) that any algebra satisfying the exchange property has bases and all the bases have the same cardinality. Thus the obvious thing to do now, in order to be able to speak about the dimension of an algebra, is to restrict our study to those algebras which satisfy the exchange property.

Matroids are usually taken to be finite, but for considering the notion of independence, finiteness is irrelevant. Infinite matroids are treated by Oxley in [33]; the following definition, which is equivalent to his, is taken from [11]. A matroid is a pair $(X,\langle\cdot\rangle)$ where $X$ is a set and $\langle\cdot\rangle: P(X) \rightarrow P(X)$ is a mapping defined on $P(X)$, the power set of $X$, and satisfying the following 
conditions $[\mathrm{C}],[\mathrm{EP}]$ and $[$ Fin]:

[C] The mapping $\langle\cdot\rangle: P(X) \rightarrow P(X)$ is a closure operator, that is,

(C1) $Y \subseteq\langle Y\rangle$, for all $Y \in P(X)$;

(C2) $Z \subseteq Y \Rightarrow\langle Z\rangle \subseteq\langle Y\rangle$, for all $Y, Z \in P(X)$;

(C3) $\langle Y\rangle=\langle\langle Y\rangle\rangle$, for all $Y \in P(X)$;

[EP] for all $x, y \in X$ and $Y \in P(X)$,

if $x \in\langle Y \cup\{y\}\rangle$ and $x \notin\langle Y\rangle$, then $y \in\langle Y \cup\{x\}\rangle$;

[Fin] for all $Y \in P(X)$, if $x \in\langle Y\rangle$, then $x \in\left\langle Y^{\prime}\right\rangle$ for some finite $Y^{\prime} \subseteq Y$.

A closure operator which satisfies [Fin] is called an algebraic closure operator. The general techniques of matroid theory guarantee, as mentioned above, that every matroid has a basis (i.e., a minimal generating set) and all the bases have the same cardinality.

Let $\mathcal{A}$ be an algebra with universe $A$. Given a subset $X$ of $A$, we denote by $\langle X\rangle$ the subalgebra of $\mathcal{A}$ generated by $X$. It is obvious that $\langle\cdot\rangle$ is an algebraic closure operator in any algebra. We say that $\mathcal{A}$ is a weak independence algebra if $(A,\langle\cdot\rangle)$ is a matroid, that is, $\langle\cdot\rangle$ satisfies [EP] (we also say that $\mathcal{A}$ satisfies $[\mathrm{EP}])$. Thus we can define the rank or dimension of a weak independence algebra to be the cardinality of any basis for the algebra.

We remark that any subalgebra of a weak independence algebra is clearly also a weak independence algebra, and hence we can also speak of the rank or dimension of a subalgebra. In particular, if $\mathcal{A}$ is a weak independence algebra and $a \in \operatorname{End}(\mathcal{A})$, then the dimension of $\operatorname{im}(a)$ is well defined. We refer to this cardinal as the $\operatorname{rank}$ of $a$, and denote it by $\operatorname{rank}(a)$.

Recall that our aim is to find a class of weak independence algebras such that for any algebra $\mathcal{A}$ in the class and any endomorphisms $a, b$ of $\mathcal{A}$, we have

$$
a \in S b S \Leftrightarrow \operatorname{rank}(a) \leqslant \operatorname{rank}(b)
$$

where $S=\operatorname{End}(\mathcal{A})$. Whether or not $(2)$ holds for a particular $\mathcal{A}$, at least $\operatorname{rank}(a)$ now has a meaning, in contrast to the situation for general algebras in (1). Thus we are going to look for conditions under which (2) holds.

To do this we need to investigate bases of subalgebras, that is, subsets of an algebra which are minimal generating sets for the subalgebras which they generate. There are several conditions involving such subsets which are equivalent to $[\mathrm{EP}]$ and which are very useful when applying $[\mathrm{EP}]$. 
Before stating these conditions we mention the following easy lemma.

Lemma 1.1. Let $Y$ be a subset of an algebra $\mathcal{A}$. Then the following are equivalent:

(1) $Y$ is a basis for the subalgebra it generates;

(2) for every $y$ in $Y$, we have $y \notin\langle Y \backslash\{y\}\rangle$.

A subset of an algebra $\mathcal{A}$ satisfying the equivalent conditions of Lemma 1.1 is said to be independent. From Exercise 6 on page 50 of [30] we have the following lemma.

Lemma 1.2. For an algebra $\mathcal{A}$, the following conditions are equivalent:

(1) $\mathcal{A}$ satisfies [EP].

(2) For every subset $X$ of $A$ and every element $u \in A$, if $X$ is independent and $u \notin\langle X\rangle$, then $X \cup\{u\}$ is independent.

(3) For every subset $X$ of $A$, if $Y$ is a maximal independent subset of $X$, then $\langle X\rangle=\langle Y\rangle$.

(4) For subsets $X, Y$ of $A$ with $Y \subseteq X$, if $Y$ is independent, then there is an independent set $Z$ with $Y \subseteq Z \subseteq X$ and $\langle Z\rangle=\langle X\rangle$.

A straightforward consequence of Lemma 1.2 is that, in a weak independence algebra, a subset is a basis if and only if it is a maximal independent subset.

We now consider equation (2) again. Let $\mathcal{A}$ be a weak independence algebra and $a, b \in S=\operatorname{End}(\mathcal{A})$. Using Lemma 1.2, it is straightforward to show that, for $a, b \in S$, if $a \in S b S$, then $\operatorname{rank}(a) \leqslant \operatorname{rank}(b)$. For the converse, we want to prove that if $\operatorname{rank}(a) \leqslant \operatorname{rank}(b)$, then there exist $u, v \in S$ such that $a=u b v$. It is easy to show that for some mappings $u^{\prime}, v^{\prime} \in T(A)$, we have $a=u^{\prime} b v^{\prime}$. However we have no guarantee that these mappings belong to $S$, that is, we do not know if we can choose these two mappings to be endomorphisms of $\mathcal{A}$.

To ensure that we can choose $u^{\prime}, v^{\prime}$ to be endomorphisms, we use the following existence condition.

[F] For any basis $X$ of $\mathcal{A}$ and a function $\alpha: X \rightarrow A$, there is an endomorphism $\bar{\alpha}$ of $\mathcal{A}$ such that $\bar{\alpha}_{\left.\right|_{X}}=\alpha$.

An algebra satisfying $[E P]$ and $[F]$ is called an independence algebra. Now, again using Lemma 1.2 , it is possible to prove that for an independence algebra $\mathcal{A}$ and $S=\operatorname{End}(\mathcal{A})$ we have

$$
a \in S b S \Leftrightarrow \operatorname{rank}(a) \leqslant \operatorname{rank}(b), \text { for all } a, b \in S .
$$


The crucial observation is that because of (4) of Lemma 1.2, condition [F] implies that any function from an independent subset of $\mathcal{A}$ can be extended to an endomorphism of $\mathcal{A}$. The details of the argument can be found in the original paper of Gould [18] where independence algebras are introduced.

We remark that there are some weak independence algebras other than independence algebras which satisfy equation (2). For example, any chain $C$ regarded as a semigroup with multiplication given by $x y=\min \{x, y\}$ is a weak independence algebra and it is known that equation (2) holds for $C$. However, if $C$ has more than one element, it is not an independence algebra.

Finally we should observe that when Gould defined independence algebras, a lot was already known about them. In contrast to the path described in this section which is based on properties of the endomorphism monoid of the algebra, the original discovery of independence algebras came through an investigation of notions of independence, as we will see in the next section. In order to avoid confusion we will use the term $C$-independence (because it is linked to a Closure Operator) for the notion of independence defined above.

\section{Thoughts of Independence in Poland}

In [28] Marczewski introduced a notion of independence (for universal algebras) and proved that many different notions of independence from numerous branches of mathematics are, in fact, particular cases of his notion.

Let $\mathcal{A}=(A, F)$ be a universal algebra ( $A$ is its universe and $F$ is the set of fundamental operations). Marczewski denoted by $A^{(n)}$ the set of all $n$-ary algebraic operations (or, following [6], the set of all $n$-ary terms). Now we say that a set $N \subseteq A$ is independent if and only if it satisfies the following condition:

$\left[M_{1}\right]$ every map $f: N \rightarrow A$ can be extended to a morphism $\phi:\langle N\rangle \rightarrow \mathcal{A}$.

(Recall that $\langle N\rangle$ denotes the subalgebra generated by $N$ ). We shall call this notion of independence $M$-independence (for Marczewski) in order to distinguish it from the $C$-independence defined in the previous section. We say that a set is $M$-dependent $(C$-dependent) if it is not $M$-independent ( $C$-independent). Investigating the interplay between these two concepts of independence led Marczewski and his colleagues to the idea of $v^{*}$-algebras [31] several decades before the advent of independence algebras [18], but in fact, the two types of algebras are precisely the same. 
After introducing $M$-independence, Marczewski provides two more characterizations of it. One is the following. A set $N \subseteq A$ is $M$-independent if it satisfies

$\left[M_{2}\right]$ for each sequence of different elements $a_{1}, \ldots, a_{n} \in N$ and for each pair of $g, h \in A^{(n)}$, if $g\left(a_{1}, \ldots, a_{n}\right)=h\left(a_{1}, \ldots, a_{n}\right)$, then $g$ and $h$ are identical in $\mathcal{A}$.

Then Marczewski closes this introductory section saying the following: If $N$ is a set of independent elements, then no $a \in N$ belongs to the subalgebra generated by the set $N \backslash\{a\}$. The converse implication is not true. With our definitions this means that in any universal algebra $M$-independence implies $C$-independence, but not conversely. Therefore we can ask what is needed so that the algebra satisfies the converse? In a vector space $M$-independence coincides with $C$-independence. Thus we can check what makes this work in a vector space to see what properties we should require a universal algebra to satisfy in order to have the two notions of independence coinciding.

Suppose that a finite set of vectors $N=\left\{x_{1}, \ldots, x_{n}\right\}$ is $M$-dependent. Then, by $\left[M_{2}\right]$, there exist two linear combinations $s\left(x_{1}, \ldots, x_{n}\right)=\sum s_{i} x_{i}$ and $t\left(x_{1}, \ldots, x_{n}\right)=\sum t_{i} x_{i}$, such that $s\left(x_{1}, \ldots, x_{n}\right)=t\left(x_{1}, \ldots, x_{n}\right)$, but $s \neq t$. Moreover, it is well known that $s \neq t$ if and only if there exists an in$\operatorname{dex} i \in\{1, \ldots, n\}$ such that $s_{i} \neq t_{i}$. (Since + is commutative we can assume without loss of generality that $\left.s_{1} \neq t_{1}\right)$. Therefore $\sum s_{i} x_{i}=\sum t_{i} x_{i}$ is equivalent to $x_{1}=\left(s_{1}-t_{1}\right)^{-1} \sum_{i=2}^{n}\left(t_{i}-s_{i}\right) x_{i}$. Thus $x_{1} \in\left\langle x_{2}, \ldots, x_{n}\right\rangle$ and hence $N$ is $C$-dependent. In general $M$-independence implies $C$-independence and now we know what is used to prove the converse in a vector space. Schematically, in a vector space, $M$-dependence implies $C$-dependence because of the following:

if $s\left(x_{1}, \ldots, x_{n}\right)=\sum s_{i} x_{i}=\sum t_{i} x_{i}=t\left(x_{1}, \ldots, x_{n}\right)$ and $s \neq t$, then

(1) there exists at least one index (say $i=1$ ) such that $s_{1} \neq t_{1}$, and

(2) there exists an $(n-1)$-term $h$ such that $s\left(x_{1}, \ldots, x_{n}\right)=t\left(x_{1}, \ldots, x_{n}\right)$ is equivalent to $x_{1}=h\left(x_{2}, \ldots, x_{n}\right)$.

Clearly the hypothesis and the second conclusion can be formulated for any universal algebra (using terms). However, the first conclusion cannot be directly expressed in a general setting. Therefore we need an equivalent way of expressing it. In fact, it is easy to see that, in a vector space $V$, if we have $s\left(x_{1}, \ldots, x_{n}\right)=\sum s_{i} x_{i}$ and $t\left(x_{1}, \ldots, x_{n}\right)=\sum t_{i} x_{i}$, then $s_{1} \neq t_{1}$ if 
and only if there exist $a_{1}^{\prime}, a_{1}, \ldots, a_{n} \in V$ such that

$s\left(a_{1}, a_{2}, \ldots, a_{n}\right)=t\left(a_{1}, a_{2}, \ldots, a_{n}\right)$ but $s\left(a_{1}^{\prime}, a_{2}, \ldots, a_{n}\right) \neq t\left(a_{1}^{\prime}, a_{2}, \ldots, a_{n}\right)$.

This fact leads to the following definition. Let $\mathcal{A}=(A, F)$ be a universal algebra and let $s, t$ be two $n$-ary terms. We say that $s$ and $t$ are distinguishable by the first variable if there exist $a_{1}^{\prime}, a_{1}, \ldots, a_{n} \in A$ such that $s\left(a_{1}, \ldots, a_{n}\right)=t\left(a_{1}, \ldots, a_{n}\right)$, but $s\left(a_{1}^{\prime}, a_{2}, \ldots, a_{n}\right) \neq t\left(a_{1}^{\prime}, a_{2}, \ldots, a_{n}\right)$. It is clear how one defines distinguishable by the ith variable

We mention that in [36], instead of saying that two terms are distinguishable by the $i$ th variable, Urbanik says that the terms depend on the ith variable.

After defining distinguishable terms (which corresponds in our scheme above to conclusion (1)), Marczewski goes on to define what he calls $v$ algebras. We say that an algebra $\mathcal{A}=(A, F)$ is a $v$-algebra, when for every pair of terms $s, t \in A^{(n)}(n=1, \ldots)$ distinguishable by the first variable there exists a term $h \in A^{(n-1)}$ such that $s\left(x_{1}, \ldots, x_{n}\right)=t\left(x_{1}, \ldots, x_{n}\right)$ is equivalent to $x_{1}=h\left(x_{2}, \ldots, x_{n}\right)$.

It is now very easy to prove that, in $v$-algebras, $M$-independence coincides with $C$-independence. After introducing $v$-algebras and proving some direct consequences of the definition, Marczewski proves the following two (easy) results.

Lemma 2.1. Let $\mathcal{A}$ be a v-algebra. Then a singleton subset of $\mathcal{A}$ whose sole element is not a constant is $M$-independent.

Lemma 2.2. If $\left\{a_{1}, \ldots, a_{n+1}\right\} \subseteq A$ is $M$-dependent, but $\left\{a_{1}, \ldots, a_{n}\right\}$ is $M$-independent, then there exists an $n$-ary term $h$ such that $a_{n+1}=$ $h\left(a_{1}, \ldots a_{n}\right)$.

After these two lemmas Marczewski proves three more auxiliary lemmas and then proves the main results of the paper. In these theorems, attention is restricted to algebras that contain some elements which are not constants.

Theorem 2.1. In a v-algebra $\mathcal{A}$ the following are equivalent:

(1) $B$ is a basis (i.e., an $M$-independent set of generators) of $\mathcal{A}$;

(2) $B$ is a minimal set of generators;

(3) $B$ is a maximal $M$-independent set.

Observe that in any algebra every $M$-independent set of generators is also a minimal set of generators and a maximal $M$-independent set. 
Theorem 2.2. If $\mathcal{A}$ is a v-algebra, then $\mathcal{A}$ admits a basis.

Theorem 2.3. If $\mathcal{A}$ is a v-algebra, then any two bases have the same number of elements.

In all the results proved after Lemma 2.2, Marczewski uses only general facts about universal algebras and Lemmas 2.1 and 2.2. Therefore it is natural to think that Marczewski was aware of this and so knew that Theorems 2.1, 2.2 and 2.3 are true for any universal algebra which satisfies Lemmas 2.1 and 2.2. Eventually in [31] Narkiewicz introduced $v^{*}$-algebras (saying that the investigation of $v^{*}$-algebras was suggested to me by Professor E. Marczewski) using the following definition.

An algebra $\mathcal{A}$ is a $v^{*}$-algebra if it satisfies the following conditions:

(I) If $a \in A$ is not a constant, then the set $\{a\}$ is $M$-independent;

(II) If $\left\{a_{1}, \ldots, a_{n}\right\}$ is an $M$-independent subset of $\mathcal{A}$, but $\left\{a_{1}, \ldots, a_{n+1}\right\}$ is not $M$-independent, then $a_{n+1} \in\left\langle a_{1}, \ldots, a_{n}\right\rangle$.

Since (I) and (II) come from Lemma 2.1 and Lemma 2.2, it follows that $v$ algebras are $v^{*}$-algebras (but not conversely). Then Narkiewicz provides an equivalent definition for $v^{*}$-algebras (suggested to him by Świerezkowski) by proving the following result.

Proposition 2.1. An algebra $\mathcal{A}$ is a $v^{*}$-algebra if and only if it satisfies the following conditions:

(III) in $\mathcal{A}, M$-independence coincides with $C$-independence;

(IV) in every subalgebra with a finite basis (i.e., $M$-independent generating set) consisting of $k$ elements, every $M$-independent set of $k$ elements forms a basis for the subalgebra.

In [32] Narkiewicz provided yet another set of defining conditions for $v^{*}$-algebras as recorded in the next result.

Proposition 2.2. An algebra is a $v^{*}$-algebra if and only if it satisfies (III) of Proposition 2.1 and $[\mathrm{EP}]$.

It is very easy to prove that (III) and [EP] are equivalent to $[\mathrm{F}]$ and [EP]. Thus the class of $v^{*}$-algebras coincides with the class of independence algebras.

It is worth observing that the research carried out in Poland during the sixties led to many other classes of algebras $\left(v_{*}\right.$-algebras, $v_{*}^{*}$-algebras, $v^{\prime}$ algebras, separable variables algebras, etc.) and to more general notions of 
independence. A comprehensive survey paper (containing more than eight hundred references) was written by Głazek [16].

\section{The Description of Independence Algebras}

As with any algebraic structure, the question arises of whether independence algebras can be classified in some way. Marczewski's paper [29] about $v$-algebras is followed in the same issue of the same journal by an announcement of Urbanik [36] in which he describes all $v$-algebras (the proofs appeared in [37]). The characterisation is up to term equivalence where, we recall, that two algebras on the same underlying set are term equivalent if their sets of $n$-ary term operations are the same for each positive integer $n$.

Characterising the $v^{*}$-algebras turned out to be much more complicated but it was eventually accomplished. For a survey paper (and proof of the final cases) see [38] to which we refer the reader for a description of all independence algebras.

It was more than 30 years before any new results about independence algebras (from the perspective of universal algebra) appeared. In [7] Cameron and Szabó prove the following result.

Theorem 3.1. The subalgebra lattice of an independence algebra of finite dimension is a Boolean lattice or a projective or affine geometry.

They also reproved the description of finite independence algebras, providing for this case an extraordinarily short proof.

\section{Independence Algebras and Semigroups}

The monoids $T(X)$ and $\operatorname{End}(V)$ (where $X$ is a set and $V$ is a vector space) have much more in common than the fact that their principal ideals form a well ordered chain. For example, if $T_{f}(X)$ denotes the set of all members of $T(X)$ with finite image, and $\operatorname{End}_{f}(V)$ denotes the set of all endomorphisms of $V$ of finite rank, then $T_{f}(X)$ and $\operatorname{End}_{f}(V)$ are both completely semisimple semigroups, that is, they are regular and all their principal factors are completely 0 -simple or completely simple. The search for algebras whose endomorphism monoids enjoy properties similar to those of $T(X)$ and $\operatorname{End}(V)$ led Gould [18] to rediscover independence algebras. In [18], she described the structure of the endomorphism monoid of an independence algebra, characterising Green's relations, and showing that the set of endomorphisms of finite rank is a completely semisimple subsemigroup. 
The original motivation for studying these endomorphism monoids was the hope of finding semigroup analogues of her work with Petrich [19] on rings of quotients. This aim has not yet been realised, but in a recent paper [13] a small step in this direction has been made.

However, since the early 1990s, endomorphism monoids of independence algebras, and related semigroups, have been extensively studied and the topic continues to receive a great deal of attention. It is not our purpose to give a comprehensive survey of this rapidly growing body of work, but it seems appropriate to give an account of some of the early results which influenced the development of the topic.

In 1966 Howie described the subsemigroup $E(X)$ of $T(X)$ generated by all the non-identity idempotents [23]. The corresponding result for $\operatorname{End}(V)$ where $V$ is a finite dimensional vector space quickly followed [10], but it was nearly twenty years later before Reynolds and Sullivan [34] found the appropriate analogue in the infinite dimensional case. Their work also uncovered a significant difference between the semigroups $E(X)$ and $E(V)$ where $X$ is an infinite set, $V$ is an infinite dimensional vector space, and where for any algebra $\mathcal{A}$ we denote the subsemigroup of $\operatorname{End}(\mathcal{A})$ generated by the non-identity idempotents by $E(\mathcal{A})$. Sullivan surveyed the parallels and distinctions between $T(X)$ and $\operatorname{End}(V)$ in an influential conference talk in 1990 which was published in [35].

Fountain and Lewin, having seen a preliminary version of Gould's paper [18], realised that independence algebras provided a suitable conceptual framework for unifying those results on products of idempotents which hold for both $T(X)$ and $\operatorname{End}(V)$. They described $E(\mathcal{A})$ for an independence algebra of finite rank in [14], proving the following result. (For a direct proof see [1].)

Theorem 4.1. If $\mathcal{A}$ is an independence algebra of finite rank $n$, then

$$
E(\mathcal{A})=\left\langle E_{1}\right\rangle=\operatorname{End}(\mathcal{A}) \backslash \operatorname{Aut}(\mathcal{A})
$$

where $E_{1}$ is the set of idempotents of rank $n-1$ in $\operatorname{End}(\mathcal{A})$.

The results of Howie [23] for finite sets and Erdos [10] are simply special cases.

Let $S$ be a semigroup generated by its set of idempotents $E$. The depth of $S$ is the smallest integer $k$ such that $E^{k}=S$. Using this notion, Howie [24] refined the result about idempotent generation of $E(X)$ when $X$ is finite by showing that $E(X)$ has depth $\left[\frac{3}{2}(n-1)\right]$. In contrast, Ballantine [5], Dawlings [9] and Laffey [27] independently obtained results showing 
that, for a vector space $V$ of finite dimension $n$, the semigroup $E(V)$ has depth $n$. Ballantine's results were extended to a special class of finite rank independence algebras in [17].

In the infinite rank case attention was restricted to strong independence algebras. An independence algebra $\mathcal{A}$ is strong when for any independent subsets $X$ and $Y$ of $\mathcal{A}$, if $\langle X\rangle \cap\langle Y\rangle=\langle\emptyset\rangle$, then $X \cup Y$ is independent. For such an algebra $\mathcal{A}$, a characterisation of $E(\mathcal{A})$ generalising the results of [23] and [34] was given in [15]. To describe this result we need the notions of shift, defect and collapse for an endomorphism of a strong independence algebra. First, we remark that the exchange property allows us to define the co-rank of a subalgebra $\mathcal{B}$ of an independence algebra $\mathcal{A}$ as follows. Let $X$ be a basis for $\mathcal{B}$ and extend $X$ to obtain a basis $X \cup Y$ for $\mathcal{A}$. Then the co-rank of $\mathcal{B}$ (in $\mathcal{A}$ ) is defined to be the cardinal $|Y|$.

We also observe that if $\alpha$ is an endomorphism of an independence algebra $\mathcal{A}$, then $\operatorname{im}(\alpha)$ and $\operatorname{fix}(\alpha)$ are subalgebras where

$$
\operatorname{fix}(\alpha)=\{a \in A: a \alpha=a\} .
$$

We can now define the defect, $d(\alpha)$, and the shift, $s(\alpha)$ as follows:

$$
\begin{aligned}
& d(\alpha)=\text { co-rank im }(\alpha), \\
& s(\alpha)=\text { co-rank fix }(\alpha) .
\end{aligned}
$$

It is a little more complicated to define the collapse of $\alpha$; we start by putting $K=\langle\emptyset\rangle \alpha^{-1}$, the inverse image under $\alpha$ of the subalgebra of constants. Now let $T$ be any subset of $A$ such that $T \alpha$ is a basis for im $\alpha$ and $\alpha$ restricted to $T$ is one-one. Let $M=\langle K \cup T\rangle$ and define the collapse, $c(\alpha)$, of $\alpha$ by

$$
c(\alpha)=\operatorname{rank} K+\operatorname{co-rank} M .
$$

This definition is independent of the choice of $T$, so we do have a well defined notion.

Now let $\mathcal{A}$ be a strong independence algebra of infinite rank. Define subsets $F$ and $Q$ of $\operatorname{End}(\mathcal{A})$ as follows:

$$
\begin{aligned}
& F=\left\{\alpha \in \text { End } \mathcal{A}: 0<d(\alpha), s(\alpha)<\aleph_{0}\right\}, \\
& Q=\left\{\alpha \in \text { End } \mathcal{A}: d(\alpha)=s(\alpha)=c(\alpha) \geqslant \aleph_{0}\right\} .
\end{aligned}
$$

In fact, it can be shown that

$$
F=\left\{\alpha \in \text { End } \mathcal{A}: 0<c(\alpha)=d(\alpha) \leqslant s(\alpha)<\aleph_{0}\right\} .
$$

Recall that an element $s$ of a monoid $S$ is unit regular if $s u s=s$ for some unit $u$ of $S$, and let $U$ be the set of unit regular members of $\operatorname{End}(\mathcal{A})$. Then 
$U$ is a submonoid of $\operatorname{End}(\mathcal{A})$ and

$$
Q=\{\alpha \in U: d(\alpha)=s(\alpha)\}
$$

We can now characterise the subsemigroup $E(\mathcal{A})$ generated by the nonidentity idempotents of $\operatorname{End}(\mathcal{A})$.

Theorem 4.2. If $\mathcal{A}$ is a strong independence algebra of infinite rank, then $F$ and $Q$ are regular idempotent generated subsemigroups of $\operatorname{End}(\mathcal{A})$ and

$$
E(\mathcal{A})=F \cup Q
$$

If $\alpha$ is an endomorphism of a vector space, then it is not difficult to see that the collapse of $\alpha$ is the nullity of $\alpha$, and this observation shows that the result of Reynolds and Sullivan [34] describing the idempotent generated subsemigroup of $\operatorname{End}(V)$ where $V$ is an infinite dimensional vector space is a consequence of Theorem 4.2. When specialised to sets, the notion of collapse we have defined is not exactly the same as the original definition in [23]. However, the two notions give the same value in the case of infinite collapse, and so the characterisation of $E(X)$ for an infinite set $X$ given in [23] also follows from Theorem 4.2.

The members of $Q$ are called balanced endomorphisms, and it is here that there is a difference betwen the set case and the vector space case. In the case of an infinite set, it was shown in [25] that the semigroup of balanced endomorphisms has depth 4 whereas in the case of an infinite dimensional vector space Reynolds and Sullivan [34] show that the depth is 3 . In [12] Fountain determined a property of some strong independence algebras which distinguishes between those for which the semigroup of balanced endomorphisms has depth 4 and those for which it has depth 3 .

We conclude by emphasising that independence algebras have proved to be very useful in providing unified proofs for analogous results for $T(X)$ and $\operatorname{End}(V)$; in explaining differences between $T(X)$ and $\operatorname{End}(V)$; and also in exporting results from semigroups to linear algebra and to some other universal algebras. (See, for example, [4], [2] and [3]).

Acknowledgements: The first author acknowledges with thanks the support of FCT, POCTI/32440/MAT/2000, and Fundação Calouste Gulbenkian.

\section{References}

1. J. Araújo, Idempotent generated endomorphisms of an independence algebra, Semigroup Forum, to appear. 
2. J. Araújo, Generators for the semigroup of endomorphisms of an independence algebra, Algebra Colloq., 9 (2002), 375-382.

3. J. Araújo, J.D. Mitchell and N. Silva, On embedding countable sets of endomorphisms, Algebra Universalis, to appear.

4. J. Araújo and F. C. Silva, Semigroups of linear endomorphisms closed under conjugation, Comm. Algebra, 28 (2000), 3679-3689.

5. C. S. Ballantine, Products of idempotent matrices, Linear Algebra and Appl. 19 (1978), 81-86.

6. S. Burris and H. P. Sankappanavar, A course in universal algebra, SpringerVerlag, New York-Heidelberg-Berlin, 1981.

7. P. J. Cameron and C. Szabó, Independence algebras, J. London Math. Soc., 61 (2000), 321-334.

8. A. H. Clifford and G. B. Preston, The algebraic theory of semigroups Vol. 1, Amer. Math. Soc., Providence, R.I., 1961.

9. R. J. H. Dawlings, Products of idempotents in the semigroup of singular endomorphisms of finite-dimensional vector space, Proc. Roy. Soc. Edinburgh Sect. A, 91 (1981/82), 123-133.

10. J.A. Erdos, On products of idempotent matrices, Glasgow Math. J. 8 (1967), 118-122.

11. C.-A. Faure and A. Frölicher, Modern projective geometry, Kluwer, Dordrecht, 2000.

12. J. Fountain, The depth of the semigroup of balanced endomorphisms, Mathematika 41 (1994), 199-208.

13. J. Fountain and V. Gould, Endomorphisms of relatively free algebras with weak exchange properties, Algebra Universalis, to appear.

14. J. Fountain and A. Lewin, Products of idempotent endomorphisms of an independence algebra of finite rank, Proc. Edinburgh Math. Soc. 35 (1992), 493-500.

15. J. Fountain and A. Lewin, Products of idempotent endomorphisms of an independence algebra of infinite rank, Math. Proc. Camb. Phil. Soc. 114 (1993), 303-319.

16. K. Głazek, Some old and new problems in the independence theory, Colloq. Math. 42 (1979), 127-189.

17. G. M. S. Gomes and J. M. Howie, Idempotent endomorphisms of an independence algebra of finite rank, Proc. Edinburgh Math. Soc. 38 (1995), 107-116.

18. V. Gould, Independence algebras, Algebra Universalis 33 (1995), 294-318.

19. V. Gould and M. Petrich, A new approach to orders in simple rings in onesided ideals, Semigroup Forum 41 (1990), 267-290.

20. G. Grätzer and J. Sichler, On the endomorphism semigroup (and category) of bounded lattices, Pacific J. Math. 35 (1970), 639-647.

21. G. Grätzer, Universal Algebra, Springer-Verlag, New York, 1979.

22. Z. Hedrlín and J. Lambek, How comprehensive is the category of semigroups?, J. Algebra 11 (1969), 195-212.

23. J. M. Howie, The subsemigroup generated by the idempotents of a full transformation semigroup, J. London Math Soc. 41 (1966), 707-716.

24. J. M. Howie, Products of idempotents in finite full transformation semi- 
groups, Proc. Roy. Soc. Edinburgh Sect. A, 86 (1980), 243-254.

25. J. M. Howie, Some subsemigroups of infinite full transformation semigroups, Proc. Roy. Soc. Edinburgh Sect. A, 88 (1981), 159-167.

26. J. M. Howie, Fundamentals of Semigroup Theory, Oxford University Press, Oxford, 1995.

27. T. J. Laffey, Products of idempotent matrices, Linear and Multilinear Algebra, 14 (1983), 309-314.

28. E. Marczewski, A general scheme of the notions of independence in mathematics, Bull. Acad. Pol. Sci. 6 (1958), 731-736.

29. E. Marczewski, Independence in some abstract algebras, Bull. Acad. Pol. Sci. 7 (1959), 611-616.

30. R.N. McKenzie, G. F. McNulty and W. F. Taylor, Algebra, lattices, varieties, Vol. I (Wadsworth, Monterey, 1983).

31. W. Narkiewicz, Independence in a certain class of abstract algebras, Fund. Math. 50 (1961/62), 333-340.

32. W. Narkiewicz, On a certain class of abstract algebras, Fund. Math. 54 (1964), 115-124.

33. J. G. Oxley, Infinite matroids, in: N. White (ed.) Matroid Applications, Cambridge University Press, 1992, pp. 73-90.

34. M. A. Reynolds and R. P. Sullivan, Products of idempotent linear transformations, Proc. Roy. Soc. Edinburgh 100A (1985), 123-138.

35. R. P. Sullivan, Transformation semigroups and linear algebra, in: T. E. Hall, P. R. Jones and J. C. Meakin (eds.), Proc. Monash Conference on Semigroup Theory, World Scientific, 1991, pp. 290-295.

36. K. Urbanik, Representation theorem for Marczewski's algebras, Bull. Acad. Pol. Sc. 7 (1959), 617-619.

37. K. Urbanik, A representation theorem for Marczewski's algebras, Fund. Math. 48 (1959/60), 147-167.

38. K. Urbanik, Linear independence in abstract algebras, Colloq. Math. 14 (1966), 233-255. 